https://helda.helsinki.fi

Brain mechanisms underlying cue-based memorizing during free viewing of movie Memento

Kauttonen, Janne

2018-05-15

Kauttonen , J , Hlushchuk , Y, Jaaskelainen , I P \& Tikka , P 2018 , ' Brain mechanisms underlying cue-based memorizing during free viewing of movie Memento ' , Neurolmage , vol. 172 , pp. 313-325 . https://doi.org/10.1016/j.neuroimage.2018.01.068

http://hdl.handle.net/10138/301556

https://doi.org/10.1016/j.neuroimage.2018.01.068

unspecified

publishedVersion

Downloaded from Helda, University of Helsinki institutional repository.

This is an electronic reprint of the original article.

This reprint may differ from the original in pagination and typographic detail.

Please cite the original version. 


\title{
Brain mechanisms underlying cue-based memorizing during free viewing of movie Memento
}

\author{
Janne Kauttonen $^{\text {a,b, }}$, Yevhen Hlushchuk ${ }^{\text {a,d }}$, Iiro P. Jääskeläinen ${ }^{\text {b }}$, Pia Tikka ${ }^{\text {a,c }}$

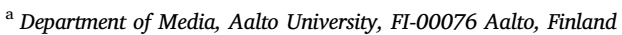 \\ ${ }^{\mathrm{b}}$ Department of Neuroscience and Biomedical Engineering, Aalto University, FI-00076 Aalto, Finland

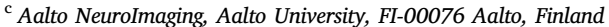 \\ ${ }^{\mathrm{d}}$ University of Helsinki and Helsinki University Hospital, HUS Medical Imaging Center, Radiology, Finland
}

\section{A R T I C L E I N F O}

\section{Keywords:}

fMRI

Naturalistic stimulus

Neurocinematics

Pattern analysis

Cued-recall

Schema

\begin{abstract}
A B S T R A C T
How does the human brain recall and connect relevant memories with unfolding events? To study this, we presented 25 healthy subjects, during functional magnetic resonance imaging, the movie 'Memento' (director C. Nolan). In this movie, scenes are presented in chronologically reverse order with certain scenes briefly overlapping previously presented scenes. Such overlapping "key-frames" serve as effective memory cues for the viewers, prompting recall of relevant memories of the previously seen scene and connecting them with the concurrent scene. We hypothesized that these repeating key-frames serve as immediate recall cues and would facilitate reconstruction of the story piece-by-piece. The chronological version of Memento, shown in a separate experiment for another group of subjects, served as a control condition. Using multivariate event-related pattern analysis method and representational similarity analysis, focal fingerprint patterns of hemodynamic activity were found to emerge during presentation of key-frame scenes. This effect was present in higher-order cortical network with regions including precuneus, angular gyrus, cingulate gyrus, as well as lateral, superior, and middle frontal gyri within frontal poles. This network was right hemispheric dominant. These distributed patterns of brain activity appear to underlie ability to recall relevant memories and connect them with ongoing events, i.e., "what goes with what" in a complex story. Given the real-life likeness of cinematic experience, these results provide new insight into how the human brain recalls, given proper cues, relevant memories to facilitate understanding and prediction of everyday life events.
\end{abstract}

\section{Introduction}

In everyday life, an event one encounters may provide a memory cue prompting interpretation of unfolding events anew from a different perspective. As if pieces of puzzle suddenly clicked together, one may foresee how the events that one is witnessing will most likely unfold. Our brains have a remarkable ability - upon a proper cue - to rapidly recall and integrate related relevant information to make sense of events, and predict what happens next. Situations like these are also common when following a movie plot. In a sense, movies are simulations of real-life events in compact form (Tikka, 2008). Memory for a complex life-like event is never a straightforward representation of the incoming information and to comprehend the sequence of unfolding real-life actions it is necessary to interpret them with reference to our prior knowledge of similar situations (Bird et al., 2015). Similarly, to follow a movie plot, the viewer must constantly recall past events in order to understand and anticipate upcoming ones (Kauttonen et al., 2014; Lerner et al., 2011).

Here, by showing our subjects the movie 'Memento' (director Christopher Nolan, 2000) during functional magnetic resonance imaging, we expected to get a step closer in revealing how human memory works in real-life situations. Specifically, Memento is a very suitably directed movie for this purpose as it contains backwards narrative structure, i.e., the story is told in reverse order starting from the causally or chronologically last event. The movie is organized so that at certain time points of overlap the viewer is cued with information that allows her/him to recall and reconstruct causal structure of previously witnessed events anew. Particularly important from our point of view is that these specific time points are audio-visually identical repeats of previously presented events. In other words, during certain exactly defined moments the ending of a subsequently presented scene is overlapping - for a few seconds - the

\footnotetext{
* Corresponding author. Laurea University of Applied Sciences, Vanha maantie 9, 02650 Espoo, Finland.

E-mail address: janne.kauttonen@gmail.com (J. Kauttonen).
} 
beginning of a previously presented scene. These audio-visually and story-wise overlapping key-events serve as temporal "bridging points" for the reconstruction of the story based on new information. In Memento, key-events work as audio-visual cues for the story. As a previously seen key-event (cue-frame; first presentation) appears the second time (keyframe; second presentation) the viewer immediately recognizes it as a repeat ("I have seen this part before") and mentally bridges, or reorganizes, the two temporally distant events as one continuous scene ("These two scenes must belong together") [See illustration in Fig. 1.]. Total 15 of such cue and key-frame event pairs, which from now on are called key-events, exist in the film. Functioning as temporal intersection points, these events encourage the viewers to update their current understanding of the plot. We expected that these temporally defined keyevents in the movie allow pinpointing the neurocognitive processes that support cue-based recall (Ezzyat and Davachi, 2011; Hayama et al., 2012; Hupbach et al., 2007; Summerfield et al., 2006) and reconstruction of events into schemas representing longer pieces of narrative.

Methodological developments have enabled use of naturalistic stimuli, such as movies, during functional magnetic resonance imaging (fMRI) of human brain blood-oxygen-level dependent (BOLD) activity (Bartels and Zeki, 2004; Chen et al., 2017; Cohen et al., 2015; Hasson et al., 2004; Jääskeläinen et al., 2008; Lahnakoski et al., 2014, 2012; Naci et al., 2014). In particular, developments in multivariate methods have allowed extraction of fine-grained information in activity patterns (Haxby, 2001; Norman et al., 2006). Multivariate approach has been applied to naturalistic stimuli, e.g., to classify movies (Emerson et al., 2015), compare perception and memory scene similarities (Bird et al., 2015; Chen et al., 2017), analyze shared response models (P.-H. Chen et al., 2015b), perform mapping between movies and annotations (Vodrahalli et al., 2017) and spatial alignment between individual brains (Guntupalli et al., 2016). Naturalistic stimuli seem to create particularly robust BOLD responses (Hasson et al., 2010), and one can use this, for example, to evoke and classify emotional states based on distributed brain activity patterns (Saarimäki et al., 2016).

Here, by using Memento as stimulus, we set forth to investigate the memory functions particularly related to cued recalling of previous events in order to make sense of the plot. For this purpose, we took advantage of the key-frames and their special role to cue the viewer's memory and reconstruction of the story, and studied if the key-frames could be associated with specific BOLD activation patterns (fingerprint patterns) at the moments they were presented. We applied event-specific pattern analysis and hypothesized that our analyses implicate brain structures that have been previously associated with long-duration memory storing and narrative comprehension. Such regions are medial temporal lobe, frontal and prefrontal cortices, hippocampus, precuneus, angular gyrus, cingulate, middle temporal pole and frontal gyri (Bird et al., 2015; Chen et al., 2017; Dehghani et al., 2017; Kauttonen et al., 2015; Nadel and Hardt, 2011; Oedekoven et al., 2017; Summerfield et al., 2006; Wheeler et al., 1995; Yaffe et al., 2014). In addition, we assumed that the right hemisphere would dominate the cognitive processes related to long-duration narrative comprehension, based on previous findings (AbdulSabur et al., 2014; Jääskeläinen et al., 2008; Marini et al., 2005; Tylén et al., 2015; Xu et al., 2005).

For a control study, we re-edited the original puzzle film version of Memento into a chronological version, and showed it to another group of subjects during fMRI. The chronological version contained the same audio-visual material as the original version, but all the scenes were rearranged according to their chronological order, and thus also without repetitive key-events. Fig. 2 depicts the timelines of both versions of Memento. Comparing the acquired fMRI control data with the original puzzle film fMRI data allowed us to separate the effect of narrative structure from those related directly to audio-visual properties of the events under scrutiny, further facilitating the interpretation of results.

We aimed to study such situations that involve three neural processes: Cued recall, schema updating and shared neural codes. Using Memento and event-related pattern analysis, we wanted to capture the very moments of these three factors co-occurring during movie viewing. It has been recently demonstrated using fMRI that during recall, originally encoded patterns (in first presentation) are reinstated in fronto-parietal regions (Bird et al., 2015; Chen et al., 2017; Oedekoven et al., 2017). These reinstatements retain information in transformed form and can be later recovered by cues (Xiao et al., 2017). Individual memories can form schemas that are high-level, dynamically evolving knowledge structures build on individual memories (Gilboa and Marlatte, 2017). They serve as general-form reference templates against which new information can be compared. Prefrontal cortex has an essential role in providing 'top-down' control to resolve the conflicts between existing memories and new events (Preston and Eichenbaum, 2013). In particular, medial prefrontal cortex is associated with conceptual knowledge integration, conceptual comprehension and assimilation of new information into a schema (Kumaran et al., 2009; Maguire et al., 1999; Mar, 2004; Schlichting and Preston, 2015; van Kesteren et al., 2014, 2013, 2010). Finally, shared neural codes correspond to neural activity patterns that remain similar across (apparently) different stimuli, thus indicating existence of conceptual similarities. Recent studies have shown existence of such shared neural codes or substrates for emotions (Saarimäki et al., 2016; Skerry and Saxe, 2014), rewards (social vs monetary; Wake and Izuma, 2017) and cognitive memory tasks (categorization vs. long-term memory; Davis et al., 2014). We are not aware of any prior studies that have considered co-occurrence of all these above factors in naturalistic setting, which is the main motivation of this work.

\section{Materials and methods}

Subjects, stimulus, annotations and data acquisition

Subjects: fMRI data from 17 right-handed healthy adults was collected, from which 13 (5 males) datasets were chosen for the final analysis. Excluded datasets included subjects that had low alertness (sleepy), missing data and/or too much motion artefacts Interestingly we also found high-pattern. The ages of the subjects were between 21 and 31 years (arithmetic mean 26 with standard deviation 3). For the chronological Memento (i.e., the control experiment), data from 14 righthanded healthy adults was collected, from which, based on the same exclusion criteria, fMRI data from 12 subjects ( 6 males) was used. The age range for this group was 20-40 years (arithmetic mean 27 with standard deviation 7).

All subjects were naive in regards to the stimuli, i.e., they reported that they had not seen the film 'Memento' previously. None of the subjects watched both versions of the movie, that is, the two subject groups

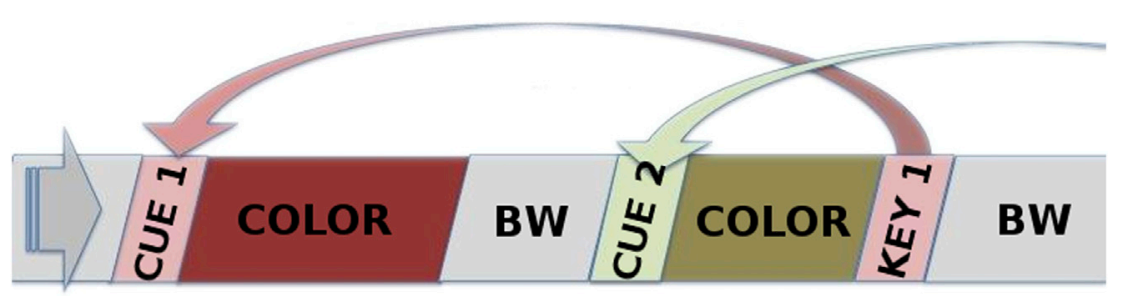

Fig. 1. (1-column, color online) Illustration of the narrative structure of Memento. The original (non-linear) version of Memento contains color and black-and-white (BW) parts with backwards structure and repeating segments. Short segments of the movie (cue-frames; CUE) are repeated later (key-frames; $\mathrm{KEY}$ ), which creates strong links (arrows) between these events. Presumably this triggers cued memory recall and updating of the subject's concurrent understanding (schema) of the plot. The movie contains 15 cue/key-frame pairs. 


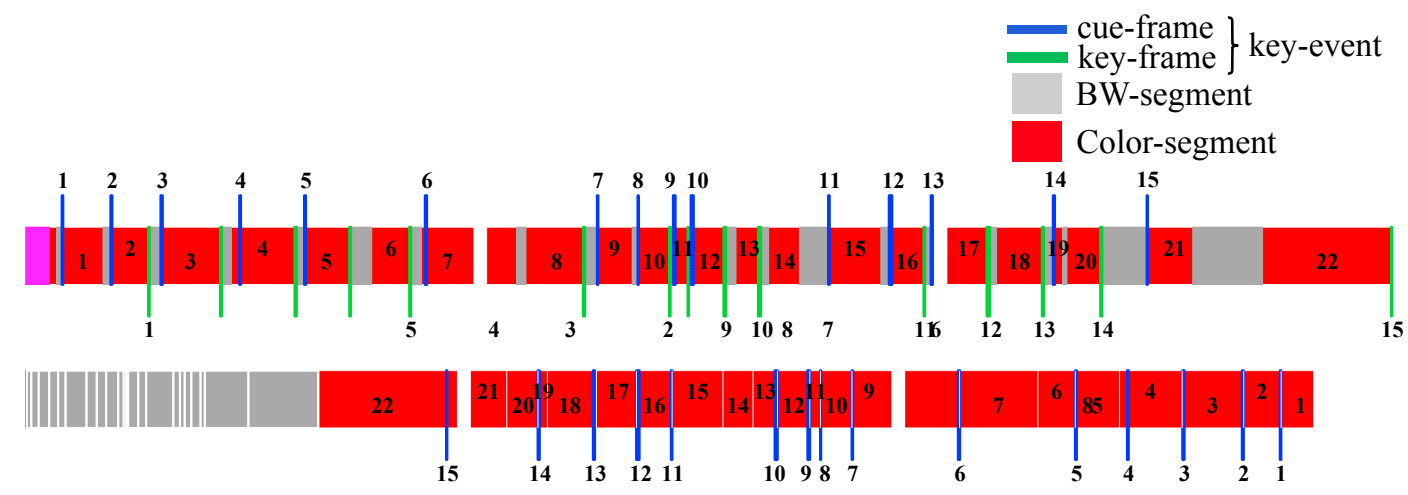

Fig. 2. (1.5-column, color online) Timelines of original (orig) and chronological (chrono) versions of 'Memento'. The movie contains 22 color (red bars with numbers 1-22) and 22 black-and-white (unlabelled grey bars) segments with 15 short clips (blue and green lines; cue and key-frames) that are pairwise audiovisually identical. Data were measured in three fMRI sessions (white bars) separated by short $(<1 \mathrm{~min})$ pauses between. Note that the chronological version is shorter than the original due to lack of redundant scene repeats and title credits (magenta bar).

were separate. The study had a prior approval by the Aalto University Ethics Committee, voluntary consent was obtained from each subject prior to participation, and we followed the principles of the Helsinki declaration throughout the study.

Stimulus: Subjects watched a 105-min long film Memento in a MRI scanner (6350s without ending credits) in three parts. The storyline contains 22 color (COL) segments that are presented in the reversed temporal order and 22 black-and-white (BW) segments in the linear, chronological order. Color and BW segments are interleaved and their storylines merge at the end of the 22nd BW segment, followed by the last color segment. In particular, movie contains 15 short clips that are each presented twice during the movie. When appearing for the first time in the film we call them cue-frames, and when they appear the second time, key-frames, cued by audio-visual content repetition. From now on, we call the corresponding 15 events, each of which has different narrative content, as key-events. Key-events had the central importance in the dataanalysis and are discussed later in greater detail. Fig. 2 depicts the timeline of the Memento, including BW and COL segments with cue and key-frames. For the control experiment, we re-arranged all 22 color and black-and-white scenes of the puzzle (non-linear) version to create a chronological (linear) version of the movie which included all 15 keyevents as a natural part of the story, however, without redundant repetition. For both versions, English subtitles beneath the movie were shown to ensure each viewer's accurate comprehension of the English language dialogue regardless of the background noise of the MRI scanner.

Data acquisition: The fMRI images were acquired with custom 30channel headcoil at MAGNETOM Skyra 3T (Siemens Healthcare, Erlangen, Germany). Functional images were obtained using a gradient echoplanar-imaging sequence with the following parameters: TR $1560 \mathrm{~ms}$, TE $30 \mathrm{~ms}$, FA $60^{\circ}, 29$ oblique axial slices, slice thickness $4 \mathrm{~mm}-4.5 \mathrm{~mm}$ with voxel sizes $3.4 \times 3.4 \times 4.0 \mathrm{~mm}$ to $3.4 \times 3.4 \times 4.5 \mathrm{~mm}$, matrix $64 \times 64$ and field of view (FOV) $22 \mathrm{~cm}$. After removal of first 8 (dummy) volumes, on average 4107 and 3883 vol per subject were collected over three sessions for the original and chronological Memento (i.e., latter one was shorted due to lack of repetitions and credits). T1-weighted anatomical images at $1 \times 1 \times 1 \mathrm{~mm}^{3}$ voxel resolution were acquired at the beginning of the first session. In addition to fMRI data, we also measured gaze direction data using EyeLink 1000 (SR Research) system with primary purpose to evaluate alertness level of subjects during scanning. The alertness was evaluated subjectively by a researcher observing the video feedback of the infrared camera. Complete eye-tracking data was obtained from 7 subjects watching the original Memento.

Key-frame annotation: Annotation of original puzzle version of Memento consisted of total 30 timestamps containing the start and end point of the cue and key-frames ( $15+15$, event IDs 1 to 15 ; see Fig. 2). For chronological version of Memento, the same 15 key-events (without repeats) were annotated. 12 out of these were also identical before the key-events appeared (i.e., parts of long identical segments; see Fig. 1), while remaining three (IDs 3, 4 and 13) had visual differences, and 10 had visually discontinuous transition at the beginning of the key-event (see Supplementary Information Appendix J for details). Timepoints were determined manually and fine-tuned up to single-frame accuracy with automated frame analysis in Matlab. Together these 15 cue-frame and key-frame pairs form the basis of key-frame model that was applied in the representational similarity analysis (RSA) of original Memento data as discussed in Section 2.3. Five of the key-frames (IDs 2, 12, 13, 14 and 15) were discontinuous in the sense that the repeating scene was discontinuous, i.e., divided in two parts with a few second gap between. In these cases, we chose the segment with the longer continuous part as the key-frame (latter part for 3 out of 5). Durations of the 15 key-event segments varied between 1.9s and 13.0s (arithmetic mean 6.4, SD 3.1). See Supplementary Information Appendix C for durations of key-events and their pairwise temporal distances.

Behavioural questionnaire: Immediately after watching Memento in the MRI scanner, all subjects filled out the post-stimulus questionnaire where we tested whether subjects were able to recall repeated scenes in Memento. Questionnaire was computerized and contained 30 color stillframes from the film. Half of these still-frames (i.e., 15) were from keyevents while remaining 15 were randomly picked from non-repeating scenes (all from color parts of the movie). The subjects had to choose if they remembered individual frames being repeated or not, and answers were collected into a binary table. We also included those three subjects ( 2 for original and 1 for chronological Memento) whose fMRI data was not included in the analysis due to excessive motion. Statistical analysis was conducted using symmetrical binomial test (i.e., chance level 0.50) to each row (subjects) and column (still-frames). Questionnaire also contained open questions about the plot and characters in order to verify alertness and general understanding of the plot. Subjects were requested to answer all questions in the form. For the chronological version of Memento in the control experiment the same questionnaire was used. As there were no repeats in this version, none of the 30 still-frames were repeated and questionnaire served only as a control. Even if the subject failed the behavioural test (i.e., did not perform above statistical significance), their fMRI data was still included in the data-analysis assuming it was otherwise valid (see Supplementary Information Appendix B).

\section{fMRI data preprocessing}

fMRI data was preprocessed using SPM12 (http://www.fil.ion.ucl.ac. uk/spm/software/spm12), FMRIB Software Library (FSL; http://fsl. fmrib.ox.ac.uk/fsl) 5.0 and in-house developed Matlab codes (http:// version.aalto.fi/gitlab/BML/bramila and http:/github.com/kauttoj/ 
fDPA_toolbox). The following preprocessing steps were applied: Slicetime correction (temporal middle-point), realignment (i.e., motion correction), anatomical and functional coregistration and normalization into MNI152 space with SPM12 segmentation. After initial preprocessing, voxel-wise time-series were detrend ${ }^{\text {ed }}$ ( 2 nd degree polynomial) and cleaned by regressing out the following 14 nuisance timeseries: 6 motion regressors ( 3 axial and 3 rotational parameters), their first derivatives (total 6) and signals from the deep white matter (5500 voxels for all subjects) and cerebrospinal fluid (up to 155 voxels depending on the subject) regions. For the latter two nuisance signals, we used first principal component for each tissue type (CompCor method, see Behzadi et al., 2007). After this, time-series were temporally high-pass filtered with $0.01 \mathrm{~Hz}$ cosine filter (SPM's spm filter). Finally, voxel-wise time-series were z-scored independently for all three sessions to remove the effect of spatial intensity variation. In order to preserve details of patterns, spatial smoothing was not applied unless stated otherwise.

Out of total 30 collected datasets $(17+13$ subjects), 25 were used in the final analysis. Omitted datasets had following issues: Drowsiness (eyes closed continuously for more than 10s; 2 subjects), technical problems (incomplete data; 2 subjects) and/or motion artefacts $(>5 \%$ bad frames reported by ArtRepair toolbox (http://cibsr.stanford.edu/ tools/human-brain-project/artrepair-software.html) with visual inspection of DVARS and framewise displacement timeseries; 1 subject).

\section{fMRI data-analysis}

We assumed that the neural functions of interest, mainly related to the cue recall, lasted up to 5 s starting from key-frame onset timepoints. In order to select proper fMRI temporal slides to extract patterns, hemodynamic lag of BOLD signal was modelled with double-gamma hemodynamic response function (HRF; SPM's spm_hrf with default parameters) with 5 s onset-to-peak delay. Using this HRF, we estimated BOLD response timeseries independently for each cue and key-frame event and normalized their maxima to 1 . Then we took mean over volumes with the estimated response over 0.5 (i.e., 50\%) per event. This resulted in four volumes that were averaged to produce one volume for each cue and keyframe. Averaging of volumes was considered necessary for three reasons: (1) it reduces the effect of timing confounds caused by inter-subject and inter-regional HRF variation as well as key-event related jitter (i.e., slice acquisition times naturally varied in respect to key-event onset times), (2) neural processes related to higher-level cognitive functions (e.g., cued recall and reasoning) likely vary between subjects and key-frames and their precise neural timing is not known, (3) averaging improves the signal-to-noise ratio of BOLD patterns (Mourão-Miranda et al., 2006). All analyses were carried out using group masks that included only voxels with valid EPI signal from all subjects in the group (either original or both original and chronological version) and were part of the loosely-thresholded grey matter tissue defined by the tissue probability template (SPM's grey.nii with threshold $>0.2$; see Supplementary Information Appendix D).

Searchlight analysis: We used volumetric searchlight with the radius of $6 \mathrm{~mm}$ containing 93 normalized voxels, which was considered suitable trade-off between pattern size and spatial specificity. In noting, using other radiuses between $4 \mathrm{~mm}$ and $8 \mathrm{~mm}$ did not change our main findings.

Representational similarity analysis: Our main method of choice for the fMRI data-analysis was representational similarity analysis (RSA). In short, RSA allows testing of hypotheses about the representational geometry of events that can be characterized by the representational dissimilarities (Walther et al., 2016). RSA models take the form of dissimilarity matrices (RDMs). We concentrated on the BOLD patterns related to the 15 key-events (including 15 occurrences of both cue- and key-frames, see Fig. 2). The hypothesized relationship between 15 cue-frame and 15 key-frame events were expressed as binary $30 \times 30$ matrices, where distance between pattern $i$ and $j$ was $1-r_{i, j}$ where $r_{i, j}$ is Pearson correlation.
We defined two model RDMs. First was the low-level model where high BOLD pattern similarity was assumed pairwise for the audio-visually identical cue- and key-frames (i.e., $r_{i, j}=1$ for matched $(i, j)$ pairs). This model was hypothesized to be relevant for the brain regions that process low-level visual and auditory information (i.e., primary visual and auditory cortices). The second model was the high-level model where high pattern similarity (a common key-frame fingerprint patterns) was assumed for all key-frames regardless of the fact that they have different low-level features (i.e., $r_{i, j}=1$ for all key-frames). This model was based on our hypothesis that underlying memory (cued recall) and narrativerelated (schema reconstruction) processing, which both can be characterized as high-level cognitive functions, do not depend on the low-level properties of the stimuli. This model specifically tests the hypothesis that key-frames are associated with a specific shared activation pattern (common code) that emerges during key-frames. In other words, the high-level model should pinpoint regions for which all key-frames (a) have similar patterns and (b) all initial cue-frames have patterns that are different from each other and different from all key-frames. The latter condition ensures that the common code - if present - is directly associated with the key-frames. Illustrations of both models are found in Supplementary Information Appendix E. RSA was performed inside searchlights where 3D spherical BOLD patterns were analyzed from all normalized voxels of the brain (Kriegeskorte et al., 2006).

We applied RSA to local BOLD patterns using overlapping searchlights covering the group mask with $174 \mathrm{k}$ normalized voxels. Since the group mask caused clipping of some searchlights at the mask borders, we required that each searchlight contained at least $50 \%$ of voxels (i.e., 47 normalized voxels for $6 \mathrm{~mm}$ radius) of the full searchlight (93 voxels). Pattern RDMs were computed from the BOLD data using Pearson correlation, resulting in empirical RDMs which were then compared against model RDMs (discussed in next paragraph) with Spearman's rank correlation (Nili et al., 2014; Schapiro et al., 2013). This resulted in subject-wise spatial correlation maps (i.e., 1st levels statistics), which were Fisher transformed and entered into group statistical test (i.e., 2nd level statistics). Computations were done using a modified version of the Matlab RSA toolbox (Nili et al., 2014). Modifications included optimizations and adding an option for permutation statistics (Mantel test for $\mathrm{RDM}$ 's). In order to test whether cue and key-frames were also associated with increase/decrease of BOLD signal levels, we also performed voxel-wise linear correlation analysis for the same timepoints that were used in the RSA pattern analysis (for details, see Supplementary Information Appendix F).

Sliding window analysis of patterns: For each subject $(s)$ and all voxels in the group mask, we computed the average correlation

$$
\overline{r_{s}}(d)=\frac{2}{N(N-1)} \sum_{j>i}^{N} r_{(i, j)}^{(s)}(d),
$$

where $N$ is the number of key-events and $r_{i, j}^{(s)}(d)$ is Pearson correlation between searchlight patterns of events $i$ and $j$ for subject $s$ with timedelay $d$ seconds (i.e., window position) before. $r_{i, j}$ :s were assumed pairwise independent and all $N(N-1) / 2$ values were used (see Supplementary Information Appendix B for details). After computing $\overline{r_{d}}$ :s for both movie versions (denoted here as $\bar{r}_{s}^{\text {(orig) }}$ and $\bar{r}_{s}^{\text {(chrono) }}$ ), we computed the mean correlation differences between two movie version data using formula

$\bar{r}(d)=\frac{1}{N} \sum_{s=1}^{N} \bar{r}_{s}^{\text {(orig) }}(d)-\frac{1}{M} \sum_{s=1}^{M} \bar{r}_{s}^{(\text {chrono })}(d)$,

where $N=12$ and $M=13$ were the number of subjects. As a result, chronological version effectively sets the baseline for the correlations and allowed us to better isolate the higher-order key-frame related neural effects (e.g., narrative comprehension and memory) by taking into account the low-level effects (e.g., camera angle and scene changes). For 
delays $d$ we used 2 s stepping and for each step we averaged three consecutive volumes (i.e., data obtained during 3.12 s window) to create patterns for analysis. Therefore delay $d=0$ s corresponds to patterns that were obtained from key-events (i.e., approximately between timepoints $0 s-3 s$ in the movie). As before, volumes were chosen at the highest HRF response locations.

Temporal delays ( $d$ 's) from -30 s to 20 s (i.e., 26 steps) were analyzed with main interest on interval -20 s to 6 s shown in Fig. 6. Positive delays $>6$ s were considered irrelevant for two reasons. Firstly, the events under scrutiny in the two Memento versions were audio-visually similar only up to the key-frames (i.e., on average up to $6 \mathrm{~s}$ ), not after them. In the original puzzle version of Memento all key-frames were followed by transition to black-and-white segments which is a large audiovisual and narrative change point (see Supplementary Information Appendix $C$ and Fig. S1). Such a strong audiovisual effect was found to create strong pattern correlations unrelated to narrative content of the movie (results not shown). For these change points we could not separate the audiovisual ("low level") and narrative effects ("high level") as they were tightly coupled. Secondly, the last key-frame (i.e., ID 15) was located at the end of the movie stimulus for the original version, i.e., no movie-related fMRI data existed after this key-frame event and related BOLD patterns could not be readily compared against other (movie-related) patterns.

After computing $\overline{r_{s}}(d)$ :s and $\bar{r}(d)$ :s for all voxels, data was analyzed in two ways. Firstly correlation maps were entered into two-sample permutation test (see below) to locate voxels with significant mean correlation difference between two movie versions. Secondly similar analysis was performed for regions of interest obtained from Harvard-Oxford MNI atlas with cerebellum parcellation (Jenkinson et al., 2012). For each subject, we averaged and Fisher transformed voxel-wise correlations over ROIs. Mean ROI-wise correlations for the original Memento were then compared against zero (one-sample $t$-test) and against those for the chronological version (two-sample $t$-test with unequal variance assumption). This analysis allowed easier inspection of region-wise temporal dynamics of correlations. Finally, as an alternative analysis, we used Power et al. (2012) spherical regions of interest (total 244) and hierarchical clustering method to study temporal organization of region-specific correlations (see Supplementary Information Appendix G for further details).

Statistical tests and analysis codes: The 2nd level group statistics for voxels was done via sign flipping (one-sample $t$-test) and group-label flipping permutations (two-sample $t$-test) implemented in FSL's Randomise tool (Winkler et al., 2014). Null hypotheses were that the mean correlation (one-sample case) and difference between means (two-sample case) for correlations between RSA model and data were not different from zero. Randomise was ran for 5.000 iterations with threshold-free cluster enhancement (TFCE) method to correct for multiple comparisons (Winkler et al., 2014). For region of interest based analyses, we used standard (parametric) one and two-sample t-tests. Analysis codes are available at http://github.com/kauttoj/memento_ draft.

Inter-subject correlation and gaze analyses: fMRI data between two Memento groups were also compared via voxel-wise intersubject correlation (ISC) analysis. We followed work by Lahnakoski et al. (2014) and applied RSA and classification methods to voxel-wise ISC values to find voxels with distinctive time courses between two Memento versions. Also, in order test if results from pattern analysis could be explained simply by gaze direction, we analyzed eye-tracking data collected from 7 subjects that watched the original Memento in MRI scanner. Details for both of these analyses can be found in Supplementary Information Appendices $\mathrm{H}$ and I.

\section{Results}

Behavioural questionnaire

14 out of all 15 subjects (12 out of 13 included in fMRI data-analysis) were able to identify repeated (key-frames) and unrepeated random stillframes from each other (uncorrected $p<0.01$, binomial test). For individual frames, 22 still-frames (with 8 key-frames) were correctly identified as being repeated or not repeated (uncorrected $p<0.05$ ). For the chronological version of Memento - where no repeats actually existed - 10 out of 13 subjects ( 9 out of 12 included in fMRI data-analysis) were able to identify that no repeating frames were present (uncorrected $p<0.05$ ). For individual still-frames 21 were correctly identified as not being repeated (uncorrected $p<0.05$ ). In conclusion, subjects were generally able to distinguish repeated and non-repeated still-frames although seven key-frames were not reliably identified by the group.

\section{Key-frame model}

Both the low and high-level RDMs resulted in statistically significant correlations in multiple cortical locations. Results are depicted in Fig. 4 (low-level model) and 5 (high-level model), with corresponding regions listed in Tables S1 and S2 in Supplementary Information Appendix A. Statistical threshold was set to $p<0.01$ with one-sample one-sided using threshold-free cluster enhancement (TFCE) multiple comparison correction algorithm (Winkler et al., 2014) which was found to closely resemblance alternative label-mixing (over rows and columns) permutation statistics at $p<0.05$ (false discovery rate (FDR; Benjamini and Hochberg, 1995) adjusted; result not shown). Label-mixing tests served as an additional control for our models. As another control for the high-level model, we replaced the cue-frames with randomly chosen events from the movie (not keys or cues, same averaging scheme), which resulted in similar results, but lacked the low-level control. Finally, we also tested an "inverse high-level model" where the identities of cue and key-frames were switched, i.e., high similarity was assumed between cue-frames, but this model revealed no significant clusters. 3D statistical maps for Figs. 3-5 are available at http://neurovault.org/collections/ 2292.

As expected, the low-level model correlated mostly with the occipital (e.g., primary visual) and parietal cortices with minor clusters in frontal and precentral gyri. Clusters were symmetrically distributed without notable lateralization. Low-level model was also associated with correlated eye-movements (see Supplementary Information Appendix H). For the high-level model the results were different as no significant correlations were in generally found in lower-level sensory regions. Highest correlations were found in superior, anterior and subcortical regions with a distinctive right hemisphere lateralization (ratio 8:3). Especially the precuneus, angular gyrus and various parts of the right frontal gyrus were highlighted. For representative examples of empirical RDMs, see Supplementary Information Appendix E. Voxel-wise signal level analysis revealed statistically significant increase in BOLD signal in parietal and frontal regions during key-frames (see Supplementary Information Appendix F).

Furthermore, in inter-subject correlation analysis, that was not limited to key-events only, differences between two movie versions were found in various frontal and parietal regions, including precuneus and angular gyrus, however, without notable lateralization (see Supplementary Information Appendix I).

\section{Sliding window pattern analysis}

In order to further test our hypothesis about the key-frame generated distinctive fingerprint pattern, we performed sliding window analysis by computing mean pattern correlation over key-frames (i.e. only the second repetition of key-events) in the original and correlating key-events in the chronological movie version. Results are depicted in Figs. 5 and 6 comparing the original version of Memento against the chronological version. Significant correlations $\left(\overline{r_{d}}\right)$ were found for several delays between -10 s and 10s. Fig. 6 depicts results for MNI region-wise averaged correlations between delays -30 s to $6 \mathrm{~s}$. Only those regions are shown that had at least one significant correlation for the original version (one- 


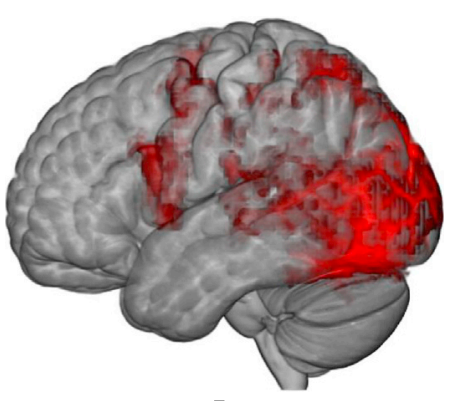

L

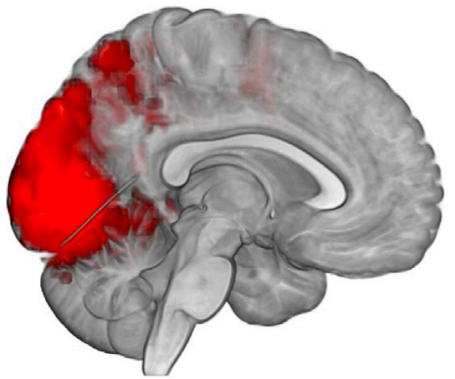

$\mathrm{L}$

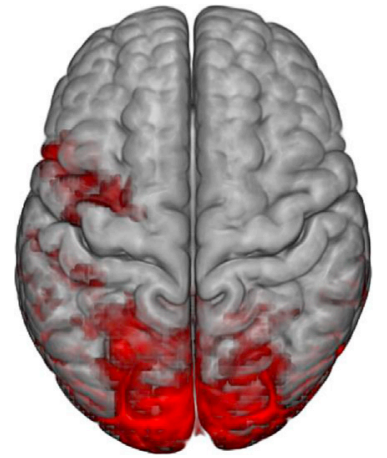

$\mathrm{R}$

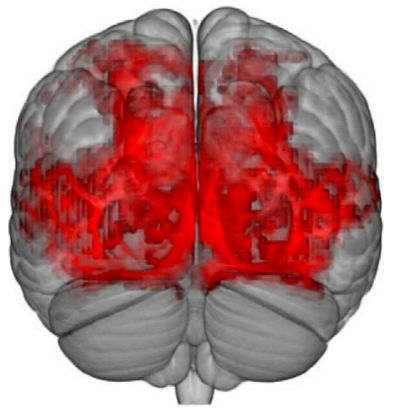

$\mathrm{R}$

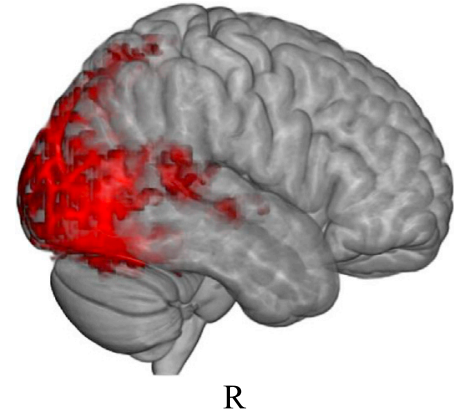

$\mathrm{R}$

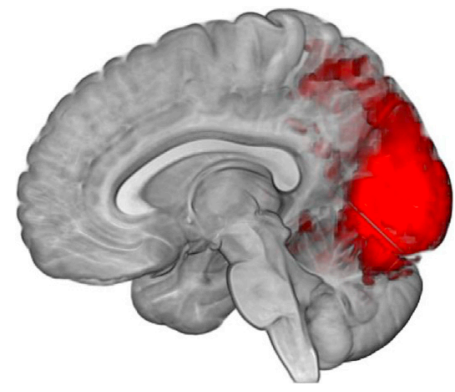

$\mathrm{R}$

Fig. 3. (2-column, color online) Result of the pattern correlation analysis with the low-level RSA model. In this model high pair-wise similarity was assumed between BOLD patterns that emerged during cue and key-frame presentation (see Supplementary Information Fig. S4a). Each colored voxel depicts a searchlight pattern centroid with significant RSA model correlation (one-sample one-sided permutation test with TFCE correction at $p<0.01$ with 13 subjects). For the listing of corresponding cortical regions, see Table S1.

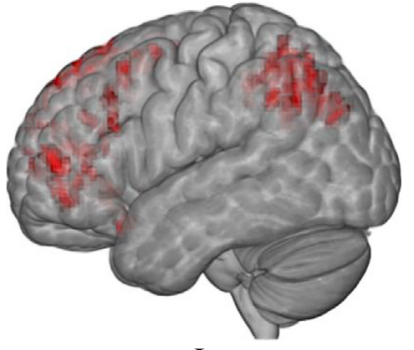

$\mathrm{L}$

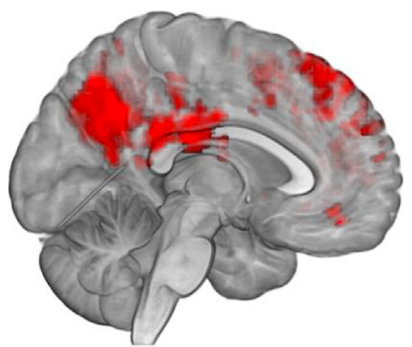

L

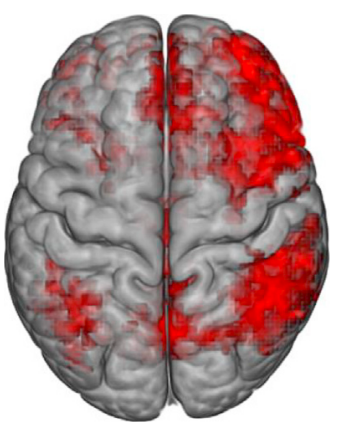

$\mathrm{R}$

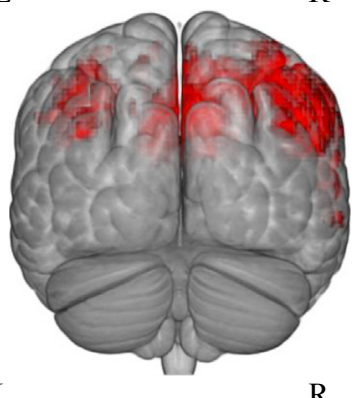

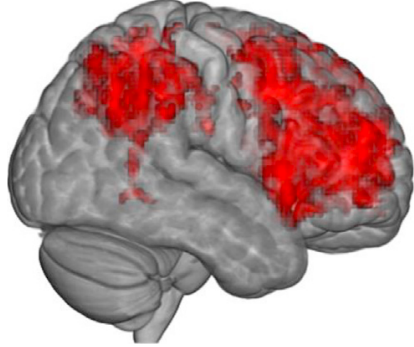

$\mathrm{R}$

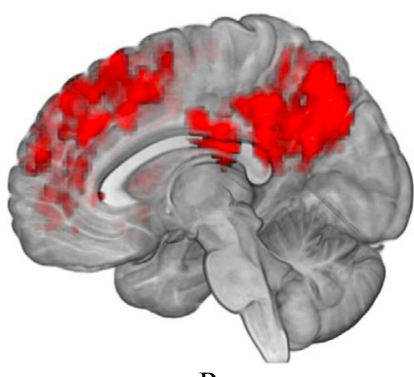

$\mathrm{R}$

Fig. 4. (2-column, color online) Result of the pattern correlation analysis with the high-level RSA model. In this model, high mutual correlation was assumed for BOLD patterns that occurred during key-frame presentation (see Supplementary Information Fig. S4b). Each colored voxel depicts a searchlight pattern centroid with significant RSA model correlation (one-sample onesided permutation test with TFCE correction at $p<0.01$ with 13 subjects). For the listing of corresponding cortical regions, see Table S2.

parietal correlations were not found for chronological Memento. There were also secondary, yet weaker correlations, before the key-frame onset time. These pre-key-frame correlations occurred for delays $-8 \mathrm{~s}$ to $-2 \mathrm{~s}$ (corresponding roughly $-7 \mathrm{~s}$ to $-1 \mathrm{~s}$ in the movie) and were mainly limited to occipital (e.g., cuneus, lingual gyrus and supracalcarine cortex) and temporal (middle and superior) cortices. Unlike key-frame related correlations, these secondary correlations emerged for both versions of the movie, being marginally larger for the original. Generality and spatial 


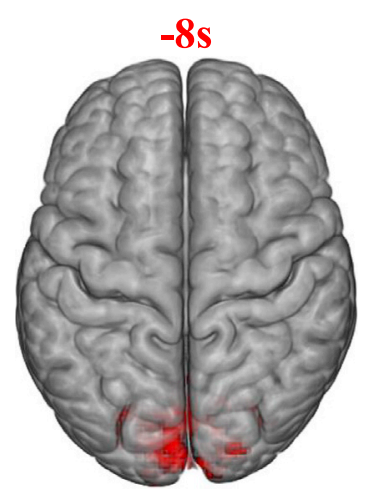

L

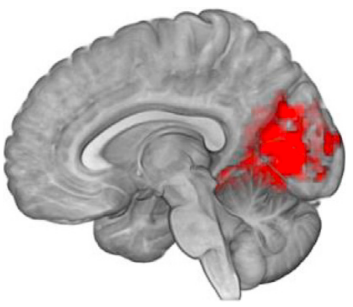

$\mathrm{R}$
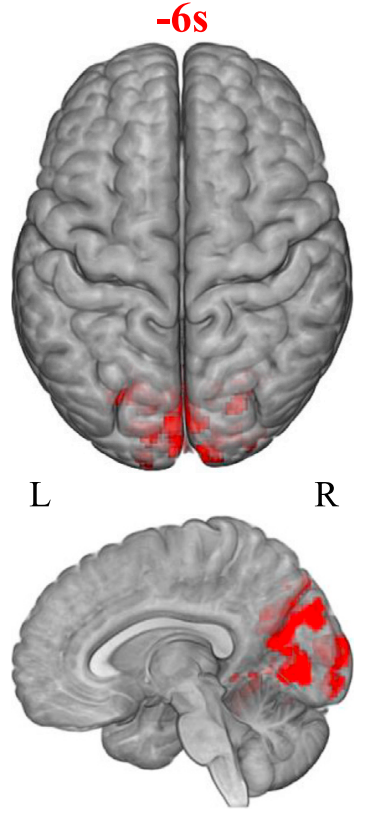

R
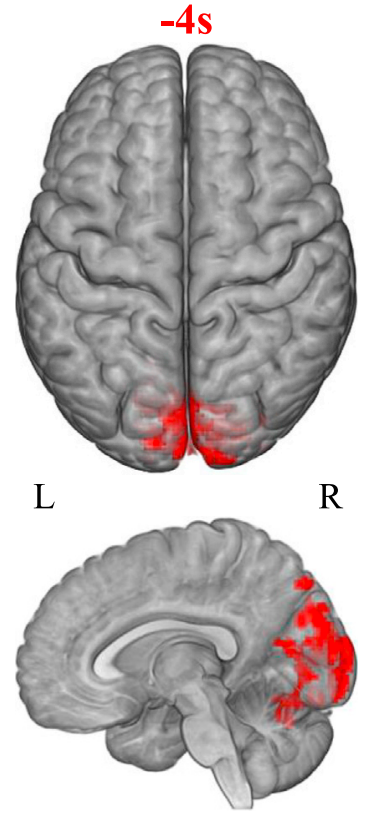

R

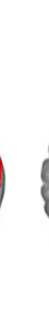

\section{key-frame onset}
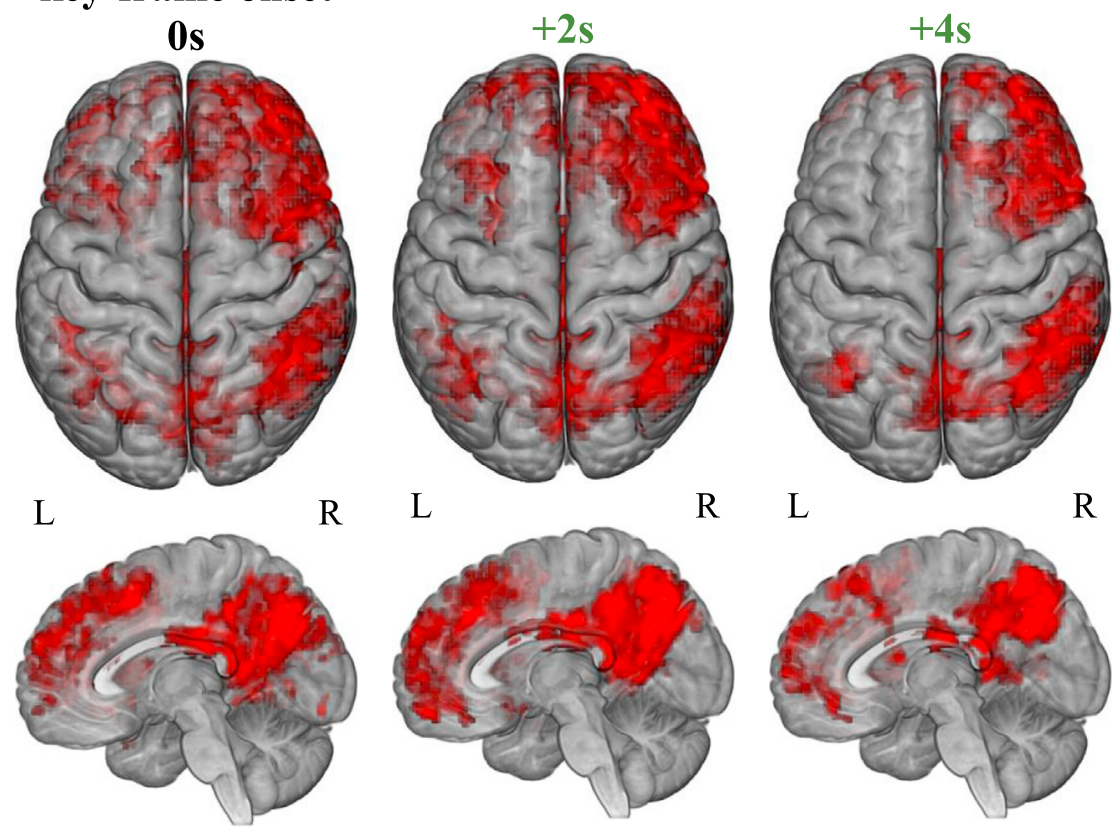

L

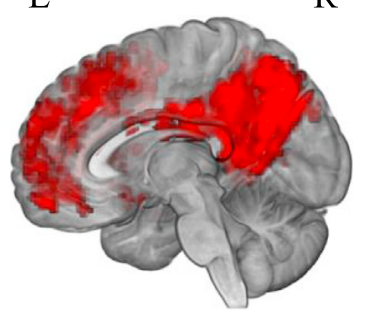

L

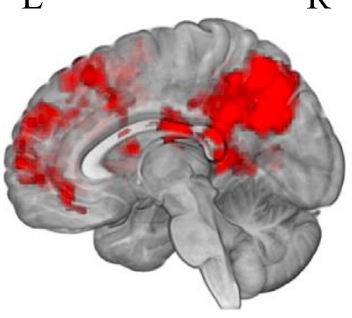

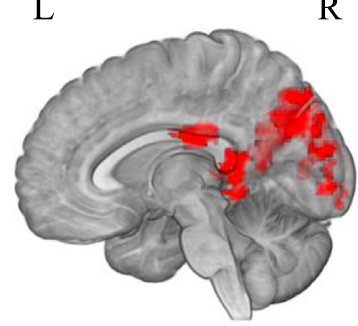

$\mathrm{R}$
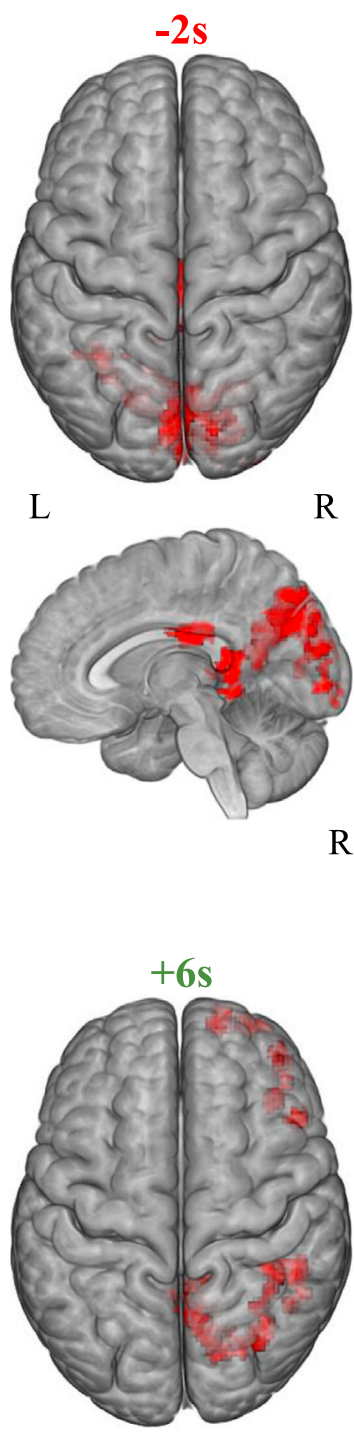

R

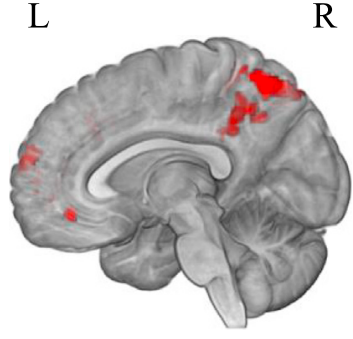

Fig. 5. (2-column, color online) Temporal dynamics of BOLD pattern correlations at the vicinity of the key-frames. Each timepoint depicts local BOLD pattern correlation between 15 time-shifted searchlight patterns $(6 \mathrm{~mm}$ searchlight with $3.12 \mathrm{~s}$ window) during Memento viewing. At $0 \mathrm{~s}$, all key-frames were temporally aligned with each other. All voxels (i.e., searchlight centroids) marked with red had significantly larger mean pattern correlation for the group viewing the original Memento compared to the control group viewing chronological Memento (control group with only cue-frames and no repetition). All eight maps were thresholded individually at $p<0.01$ (two-sample one-sided permutation $t$-test for $13+12$ subjects, TFCE corrected).

distribution of these correlations suggest that they were likely related to low-level cinematic properties of the stimulus, such as scene transition and camera framing changes. This was indeed found to be general phenomena and similar occipital cortex -centralized correlations were also found for other timepoints with similar low-level cinematic changes (data not shown). In another, ROI-based analysis, temporal clustering analysis revealed separation between occipital-temporal and frontoparietal regions with corresponding correlation peaks around $-4 \mathrm{~s}$ and 2s (see Supplementary Information Appendix G).

\section{Discussion}

In this work we used fMRI to study the neural basis of real-time reconstruction of one's understanding of a continuously unfolding story when presented with key memory cues during free-viewing of full-length movie Memento that has a unique temporally nonlinear narrative. We specifically analyzed high-level neural functions that could be associated with specific key-event repetitions (cue- and key-frames) in the movie. We hypothesized that recognition of a repeated key-event (key-frame) would prompt recall of relevant memories of the narrative events 


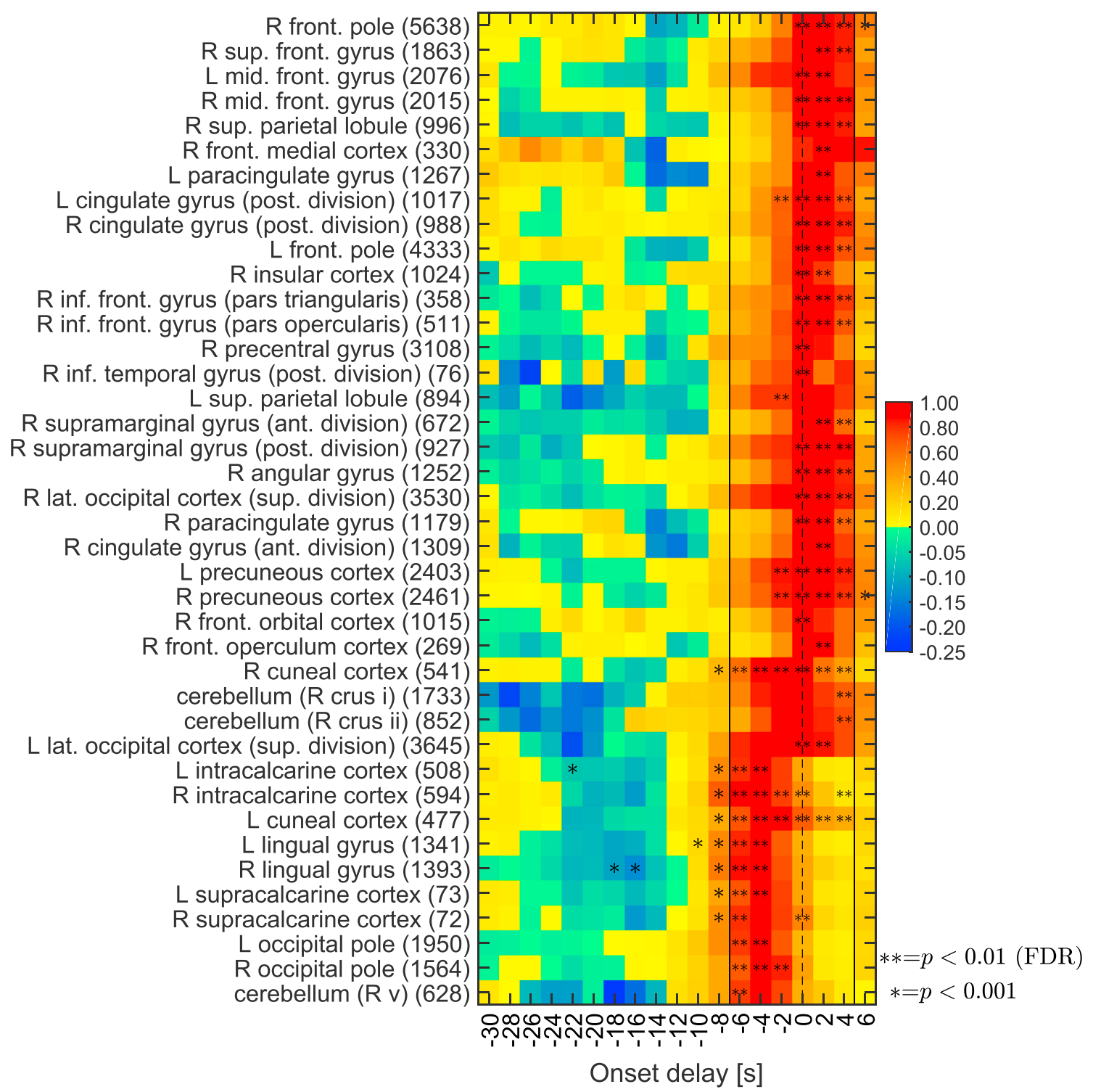

Fig. 6. (1.5-column, color online) Temporal dynamics of BOLD averaged pattern correlations at the vicinity of the key-frames for cortical regions-of-interest (ROIs). Averaging was performed using Harvard-Oxford atlas. Each colored element depicts a mean pattern correlation between 15 (time-shifted) key-frame events, all normalized voxels (count shown in parenthesis) inside the region and over subjects. Regions are arranged according to peak correlation time. All 40 (12 in the left hemisphere) shown regions had at least one significant peak when compared against zero (one-sample $t$-test for 13 subjects) and against the control group (chronological Memento viewers; two-sample $t$-test for $13+12$ subjects; both tests two-sided at $p<0.01$ FDR adjusted) between onset delays $-6 \mathrm{~s}$ and $4 \mathrm{~s}$ (black vertical lines). Mean correlation values were scaled ROI-wise (rows) with their maximum absolute values for easier visual comparison. Results for ungrouped voxels are shown in Fig. 5 for delays $-8 \mathrm{~s}$ to $+6 \mathrm{~s}$.

enabling connecting of the earlier scene with the on-going scene, thus facilitating understanding of the story piece-by-piece. We hypothesized that this would engage co-occurrence of cued-recall and schemaupdating neural activity patterns in the brain. To our knowledge, this is the first time these processes are studied together using long-duration naturalistic stimulus. We used event-related pattern analysis to disclose extended "neural fingerprint pattern" network containing various higherlevel anterior and posterior cortical regions with notable righthemisphere lateralization. Our results shed light on what happens in the brain during fast-paced cued recall and schema updating in real-life like situations. In the following, we will discuss our findings in detail.

\section{Low and high-level models}

The low-level model served as a starting point for the pattern analyses. This model was designed to pinpoint brain regions with similar activation patterns between paired cue- and key-frames. We expected to see activity in the primary sensory regions that process the low-level properties of the movie. This was indeed found to be the case as the model resulted in high correlations mainly in the occipital and parietal cortices (see Fig. 3). Further, the eye-gaze fixation patterns were highly correlated during the presentation of the same clips (see Supplementary Information Appendix H). We also expected higher correlations in the primary auditory cortices, but this was not the case probably due to fact that the key-events were short (from 2 s to $13 \mathrm{~s}$ ) and contained rather minimal auditory stimulation. This was also the case with the high-level model.

Next, we tested our main hypothesis with the high-level model, where we assumed that key-frames would activate neural processes associated with cued recall and reconstruction/updating of schema of the story. If this was the case, there should be common neural fingerprint patterns in the BOLD signal similarly for all key-frame moments. Indeed, the high- 
level model revealed network of voxels with high correlations in various higher-order (non-sensory) cortical and subcortical regions. These included precuneus, angular gyrus (ANG), lateral occipital cortex (LOC), cingulate gyrus (CG), as well as lateral, superior gyrus (LSG), and middle frontal gyrus (MFG) within frontal poles (see Fig. 4). These regions have large overlap with the well-known default mode network (DMN; Gusnard and Raichle, 2001) and also fronto-parietal control (Vincent et al., 2008) and core recollection (Thakral et al., 2015) networks. DMN includes mainly median temporal lobe (MTL), medial prefrontal cortex (mPFC), posterior CG and precuneus. Core recollection network includes regions that are consistently co-activated in association with successful recollection (King-Casas et al., 2005; Rugg and Vilberg, 2013): ANG, mPFC, precuneus, hippocampus, parahippocampal cortex (PHC) and MTG. Fronto-parietal control network includes $\mathrm{mPFC}$, intra-parietal sulcus (IPS), anterior insula and dorsal precuneus.

\section{Key-frame effect and neural fingerprint patterns}

Our result for the high-level model indicates that a common neural process, or a set of simultaneous processes, is executed during keyframes. This was confirmed with alternative sliding window analysis where we compared pattern correlations against those obtained for chronological version of the movie (see Figs. 5 and 6). Importantly, the key-frame effect did not depend on low-level audio-visual properties of the stimulus, since these properties were highly dissimilar across the keyframes. Further, as the key-events are not audio-visually related, the keyframe effect cannot be simply reactivation (i.e., reinstatement) of neural patterns of cue-frames (encoding phase). Note that these fingerprint patterns need not to be limited to key-frames only and can occur at other (subject-specific) timepoints during movie as new information is revealed. However, we were only able to study key-frames as they were time-locked between all subjects. We argue that key-frame effect and related neural fingerprint patterns represent a common neural code similar to those found for emotions (Saarimäki et al., 2016; Skerry and Saxe, 2014), rewards (Wake and Izuma, 2017) and cognitive memory tasks (Davis et al., 2014). Recently Richter et al. (2016) studied fMRI activity patterns and found evidence that memory integration processing state is qualitatively distinct from encoding and retrieval. They further showed that this memory integration state was reflected in broadly distributed neural activity patterns containing both frontal and parietal regions. We argue that our key-frame effect is related to this integration and originates from a novel combination of cued recall and story-related schema reconstruction. Next, we discuss these two factors in more detail.

\section{Key-frame effect and cued recall}

Memory traces are stored in overlapping and widely distributed networks and all cortical regions have property of storing information with varying temporal lengths (Fuster, 1997; Hasson et al., 2015). Previous studies associated to cued recall highlight medial frontal gyrus, posterior CG and MTG (Polyn et al., 2005) and, more generally, structures of the DMN (Rugg and Vilberg, 2013). ANG, precuneus, posterior CG and $\mathrm{mPFC}$ have been in associated in the encoding and retrieval of episodic memories and in a variety of high-level cognitive processes, e.g., decision making (Kim, 2010; King-Casas et al., 2005; Rugg and Vilberg, 2013; Tomlin et al., 2006). Precuneus and medial prefrontal regions appear to store context dependent information (Ames et al., 2015). Precuneus, frontal gyrus (medial, inferior and superior parts) and angular gyrus have been linked to recollection of previously seen film clips (St-Laurent et al., 2015).

PFC tends to be more activated especially for recognition memory tasks. For example, in picture-based memory tasks middle-dorsolateral PFC has been found to monitor familiarity without need for repeated items to be identical (Schon et al., 2013). In a meta-analysis by Kim (2013) it was found that cognitive-control network regions (incl. dorsolateral and dorsomedial PFC and bilateral intraparietal sulcus) showed greater activation in conditions associated with greater demand for controlled memory retrieval processing. It has been proposed that posterior parietal cortex, particularly ANG, is responsive for retrieved of information, perhaps by accumulating or temporarily maintaining the information (Hayama et al., 2012; Vilberg and Rugg, 2008). The medial temporal lobe has not only been found to be involved in the encoding and retrieval of past events, but also in the deliberate imagination of future events (Addis et al., 2007; Hassabis and Maguire, 2007). Thakral et al. (2017) localized recollection to ANG, MTG, posterior parietal cortex (PCC) and dorso-lateral PFC and also reported that there was no difference between strong and weak memories, indicating that recall - at least in these areas - is largely an automated process. In a study by Kuhl and Chun (2014) it was found that in MFG, mPFC, supramarginal gyrus and ANG reactivation reflected similarity between a cue word and associated picture that had no perceptual overlap.

For the current study, the strongest evidence comes from other neurocinematic studies. Recently Chen et al. (2017) showed 50-min movie in fMRI and found that free recall reactivated patterns in DMN, including posterior medial cortex (PMC), $\mathrm{mPFC}$, PHC and PPC. There reactivation patterns were shared between subjects, suggesting systematic and generic representation transformation of memories (i.e., schema construction). In a related study, cued recall memory reinstatement was similarly found in precuneus, inferior lateral parietal lobe, ANG, MTG and middle occipital gyrus (Oedekoven et al., 2017).

\section{Key-frame effect and schemas}

In order to follow a plot in a movie, it is not enough to remember individual events, but one also needs to relate them to each other. Here, we call such higher-level structured memory representations schema models (McKenzie and Eichenbaum, 2011; Preston and Eichenbaum, 2013). According to this model, new memories are assimilated into neocortical memory networks (schemas) through elaboration and modification of the network structure. Consolidation of incoming memories occurs by integrating them into active, pre-existing memories via reorganization (schema modification) of common elements within the cortex and hippocampus (McKenzie and Eichenbaum, 2011; Schlichting and Preston, 2015). In this context, PFC has an essential role in providing 'top-down' control to resolve the conflicts between existing memories and new events (Preston and Eichenbaum, 2013). Successful encoding of incoming schema-related information is associated with enhanced mPFC activity (van Kesteren et al., 2014).

It has been proposed that mPFC is the hub of a network that is implicated in assimilating recently acquired information, initially dependent on hippocampus, to pre-existing schemas, which can then be used to recover those memories independently of the hippocampus (Sharon et al., 2011). Consistent with this notion, van Kesteren et al. (2013) showed that medial prefrontal activity is predictive of enhanced memory for congruent information, which presumably is integrated into a pre-existing schema. mPFC then helps to integrate distinct elements of memories, which can be abstracted across events and experiences, a necessary condition for schema-based encoding (Schlichting et al., 2015). mPFC has been associated with various goal-oriented behavioural functions, may influence memory integration by biasing reactivation toward those memories that are most relevant for the on-going plot. These concepts suit well in the free-viewing situation in our study.

\section{Key-frame effect and DMN}

A common factor for fMRI studies applying long-duration naturalistic stimulus appear to be involvement of DMN, particularly precuneus and ANG (Chen et al., 2017; J. Chen et al., 2015a; Dehghani et al., 2017; Hasson et al., 2004; Jääskeläinen et al., 2008; Kauppi et al., 2010; Lerner et al., 2011). Precuneus is known as a functional core of the DMN (Utevsky et al., 2014). Together with cingulate cortex it can be considered as part of a neural system linked to narrative comprehension 
(Whitney et al., 2009). It has been suggested that ANG serves as a "convergence zone" for formation of complex, multi-domain representations assembled out of lower-level representations that are distributed across multiple modality- and domain-specific cortical region (Rugg and King, 2017). Furthermore, DMN regions (including posterior medial cortex, MPFC, MTG, and ANG) have been identified as having particularly long processing timescales (J. Chen et al., 2015a; Hasson et al., 2015; Lerner et al., 2011; Tylén et al., 2015) and they are involved in large number of tasks including episodic memory recollection, decision making, prospective thinking and schema knowledge (Binder et al., 2009; Maguire et al., 1999; Mar, 2004; Price, 2012; Rugg and Vilberg, 2013; van Kesteren et al., 2010). These findings suggest that DMN carries information about high-level structure in the world that provides a schematic context over individual events (J. Chen et al., 2015a). Therefore, it's not surprising that DMN was also strongly present in our fingerprint patterns.

\section{Lateralization of fingerprint patterns}

Our results for the key-frame effect indicated notable right lateralization of the fingerprint patterns, particularly on the frontal regions. This indicates the need for semantic integration of the story, which was one of our key hypotheses behind the high-level model. This is supported by the previous findings for narrative comprehension (AbdulSabur et al., 2014). Right hemisphere appears to dominate in discourse processing (Marini et al., 2005; St George et al., 1999), or broader inference for natural language (Jung-Beeman, 2005; Xu et al., 2005). Updating one's understanding of the plot (schema) requires more cognitive effort than simple recall (Tylén et al., 2015). On the other hand, majority of human memory studies that use non-naturalistic stimuli have reported dominance of the left hemisphere (Johnson et al., 2013; Kim, 2011; Scalici et al., 2017), while for studies using longer-duration, naturalistic stimuli results are bilateral or right dominant (Chen et al., 2017; J. Chen et al., 2015a; Dehghani et al., 2017; Hasson et al., 2004; Jääskeläinen et al., 2008; Kauppi et al., 2010; Lerner et al., 2011). Based on the hemispheric encoding/retrieval asymmetry model and supported by various neuroimaging studies, right PFC is typically engaged more than left in memory retrieval processes (Habib et al., 2003; Tulving et al., 1994). Our result supports the view that fingerprint patterns do not reflect simple cued recall effect, but instead a simultaneous cued recall and integration during the rapid-pace narrative processing.

\section{Synchronization differences and temporal dynamics of pattern correlations}

Differences in brain activity between two versions of Memento were not limited to those related to key-events. In our supporting inter-subject correlation analysis, we found that two versions of the Memento resulted in distinctive temporal BOLD activations between groups in widely distributed cortical regions overlapping DMN (see Supplementary Information Appendix I). These results show that temporal order has a fundamental effect on how these movies are processed in the brain and differences are present both very short (few seconds for key-frames) and long (minutes for complete segments) temporal scales. This is hardly surprising given differences in cognitive effort when viewing the original vs. the chronological version of the movie, as there was no need to connect the different scenes via key-frames during the latter.

Purpose of the sliding window analysis was two-fold. Firstly, it did not rely on RSA analysis and allowed comparison against chronological Memento data obtained from independent group of subjects. Secondly, it allowed scrutinizing temporal dynamics of pattern correlations, which pinpointed the key-frame effect also temporally. Interestingly we also found high-pattern correlations already some seconds before onset of key-frames in occipital-visual regions (see Fig. 5). This effect was observed for both movie versions. The most likely explanation for this effect is the camera framing and scene changes that were synchronized as a side-product when synchronizing key-frames (see Supplementary Information Appendix I). It has been found that scene transitions work as effective modulators of BOLD signal (Lu et al., 2016). This view is supported by the fact that related regions are all in lower-level visual regions. On the other hand, anticipation of upcoming key-events could be also involved as the movie scenes follow each other in logical manner and are not random. In a study by Polyn et al. (2005) such anticipation effect was found in the free-recall of items task, when category-specific patterns of activity emerged around $6 \mathrm{~s}$ prior to verbal recall from a given category, indicating anticipation.

\section{Possible confounds and limitations}

There could be also other, simpler, factors that contribute to the keyframe effect. If we neglect the fact that the key-frames belong to an ongoing movie and instead treat them simply as stimulus repeats, we may assume that repetition suppression/adaptation takes place (Grill-Spector et al., 2006; Segaert et al., 2013). Repeated and expected stimuli tend to result in weaker BOLD responses compared to novel or unexpected ones (Grotheer and Kovács, 2016; Segaert et al., 2013). Notably, in our univariate analysis we found activity decrease only in occipital cortex (primary visual), which was not part of the key-frame fingerprint patterns. In other words, we found no evidence for notable repetition suppression effect in areas involved in the key-frame pattern. We, however, found signal increase in various higher-level regions that partially overlapped our fingerprint patterns (see Fig. S6). This phenomenon is known as repetition-enhancement effect and has been found in, e.g., PFC, IPS, IPL and MTG (see Fig. 1 in Segaert et al., 2013). The key-frames are not simple stimulus repeats, but carry relevant information of the story (intersection points) and can be considered conceptually novel. This can effectively nullify the classical repetition-suppression effect (Reggev et al., 2016). Furthermore, the repetition interval is an important factor: Suppression is prominent only for relatively rapid repeats (less than a minute) and can turn into enhancement with longer inter-repeat delays of several minutes, like in our study. This type of enhancement has been found in the precuneus, posterior cingulate and (right) dorsolateral PFC (Bradley et al., 2015), which also turn up in our key-frame fingerprint patterns. Various factors can lead to enhancement, such as novel network formation, selective attention and additional cognitive processing (Segaert et al., 2013). Given the overlap between areas previously found to exhibit repetition-enhancement and the key-frame patterns in the present study, repetition-enhancement could be one of the mechanisms responsible for the key-frame effect.

At the same time, the main strengths and main limitations of this work comes from our decision to use movie stimulus and uninterrupted free-viewing design. Having the real-life like conditions can be seen as a strength, yet as limitations, firstly, we were unable to look into specific coupling mechanisms of cued recall and schema reconstruction, i.e., are they equally responsive for the fingerprint pattern or does one dominate over the other in the process and in what specific circumstances. Secondly, we were unable to collect behavioural data, such as details of keyframe events, during measurement. Thirdly, the number of key-events was relatively small (15) compared to the length of the stimulus (2h), which was not particularly efficient and did not allow us to use more sophisticated analysis (e.g., classification). Furthermore, as all cue and key-frames were short-duration and treated separately in our analysis (i.e., in single-trial fashion), we could take no advantage of multiple repeat design and GLM to estimate responses of voxels. Instead we used fMRI data directly with averaging approach. However, as the long and complex narrative was an essential part of our study, no simple alternatives existed; if more controlled (artificial) stimulus is used one may deviate too far from real-life-like setting. This remains a challenge for the future studies. 


\section{Conclusions}

Our results show that cognitive functions related to memory and narrative processing are reliably activated across viewers and can be temporally pinpointed to specific key-frame events in the narrative in free-viewing conditions. This was made possible by taking advantage of the nonlinear story structure with repeating story segments (key-frames) in movie Memento and using event-related pattern analysis technique. We were able to associate key-frames with a common "neural fingerprint" activity patterns. This network covered various frontal, higherorder parietal and subcortical regions, mainly precuneus, angular gyrus, cingulate gyrus and frontal pole with right hemispheric lateralization bias. We argue that the main process driving these regions was memory processing, especially cued recall, and followed by rapid reconstruction of narrative schema. Novelty of our study comes from combining a continuous movie stimulus with build-in repeats with eventrelated pattern analysis. Our results give insight to neural processing during moments of real-time reconstruction of one's understanding of a continuously unfolding narrative at the moment of memory cues in lifelike setting. In future, it would be interesting to test if our key-frame effect can be reproduced with tailored stimuli that would also allow careful manipulation and measurement of relative weights between recall and schema update effects.

\section{Acknowledgments}

We thank the film editor Jelena Rosic for annotating and editing 'Memento' and Marita Kattelus for assistance in the fMRI scanning. We also thank Uri Hasson for valuable comments. The fMRI data was measured at the AMI Centre Aalto NeuroImaging and computational resources were provided by Science-IT, Aalto University School of Science. The research was funded by aivoAALTO and Aalto Starting Grant for NeuroCine at the Aalto University.

\section{Appendix A. Supplementary data}

Supplementary data related to this article can be found at https://doi. org/10.1016/j.neuroimage.2018.01.068.

\section{References}

AbdulSabur, N.Y., Xu, Y., Liu, S., Chow, H.M., Baxter, M., Carson, J., Braun, A.R., 2014 Neural correlates and network connectivity underlying narrative production and comprehension: a combined fMRI and PET study. Cortex 57, 107-127. https:// doi.org/10.1016/j.cortex.2014.01.017.

Addis, D.R., Wong, A.T., Schacter, D.L., 2007. Remembering the past and imagining the future: common and distinct neural substrates during event construction and elaboration. Neuropsychologia 45, 1363-1377. https://doi.org/10.1016/ j.neuropsychologia.2006.10.016.

Ames, D.L., Honey, C.J., Chow, M.A., Todorov, A., Hasson, U., 2015. Contextual alignment of cognitive and neural dynamics. J. Cogn. Neurosci 27, 655-664. https:// doi.org/10.1162/jocn_a_00728.

Bartels, A., Zeki, S., 2004. Functional brain mapping during free viewing of natural scenes. Hum. Brain Mapp. 21, 75-85. https://doi.org/10.1002/hbm.10153.

Behzadi, Y., Restom, K., Liau, J., Liu, T.T., 2007. A component based noise correction method (CompCor) for BOLD and perfusion based fMRI. Neuroimage 37, 90-101. https://doi.org/10.1016/j.neuroimage.2007.04.042.

Benjamini, Y., Hochberg, Y., 1995. Controlling the false discovery rate: a practical and powerful approach to multiple testing. J. R. Stat. Soc. (Series B) 57, 289-300.

Binder, J.R., Desai, R.H., Graves, W.W., Conant, L.L., 2009. Where is the semantic system? A critical review and meta-analysis of 120 functional neuroimaging studies. Cereb. Cortex 19, 2767-2796. https://doi.org/10.1093/cercor/bhp055.

Bird, C.M., Keidel, J.L., Ing, L.P., Horner, A.J., Burgess, N., 2015. Consolidation of complex events via reinstatement in posterior cingulate cortex. J. Neurosci. 35, 14426-14434. https://doi.org/10.1523/JNEUROSCI.1774-15.2015.

Bradley, M.M., Costa, V.D., Ferrari, V., Codispoti, M., Fitzsimmons, J.R., Lang, P.J., 2015 Imaging distributed and massed repetitions of natural scenes: spontaneous retrieval and maintenance. Hum. Brain Mapp. 36, 1381-1392. https://doi.org/10.1002/ hbm. 22708 .

Chen, J., Honey, C.J., Simony, E., Arcaro, M.J., Norman, K.A., Hasson, U., 2015a Accessing real-life episodic information from minutes versus hours earlier modulates hippocampal and high-order cortical dynamics. Cerebr. Cortex 26. https://doi.org/ 10.1093/cercor/bhv155 bhv155.
Chen, P.-H., Chen, J., Yeshurun, Y., Hasson, U., Haxby, J., Ramadge, P.J., 2015b. A reduced-dimension fMRI shared response model. In: Neural Information Processing Systems Conference (NIPS). pp. 460-468.

Chen, J., Leong, Y.C., Honey, C.J., Yong, C.H., Norman, K.A., Hasson, U., 2017. Shared memories reveal shared structure in neural activity across individuals. Nat. Neurosci. 20, 115-125. https://doi.org/10.1038/nn.4450.

Cohen, A.-L., Shavalian, E., Rube, M., 2015. The power of the picture: how narrative film captures attention and disrupts goal pursuit. PLoS One 10, e0144493. https:// doi.org/10.1371/journal.pone.0144493.

Davis, T., Xue, G., Love, B.C., Preston, A.R., Poldrack, R.A., 2014. Global neural pattern similarity as a common basis for categorization and recognition memory, 34, 7472-7484. https://doi.org/10.1523/JNEUROSCI.3376-13.2014.

Dehghani, M., Boghrati, R., Man, K., Hoover, J., Gimbel, S.I., Vaswani, A., Zevin, J.D., Immordino-Yang, M.H., Gordon, A.S., Damasio, A., Kaplan, J.T., 2017. Decoding the neural representation of story meanings across languages. Hum. Brain Mapp. 38, 6096-6106. https://doi.org/10.1002/hbm.23814.

Emerson, R.W., Short, S.J., Lin, W., Gilmore, J.H., Gao, W., 2015. Network-level connectivity dynamics of movie watching in 6-year-old children. Front. Hum. Neurosci. 9, 1-8. https://doi.org/10.3389/fnhum.2015.00631.

Ezzyat, Y., Davachi, L., 2011. What constitutes an episode in episodic memory? Psychol. Sci. a J. Am. Psychol. Soc./APS 22, 243-252. https://doi.org/10.1177/ 0956797610393742 .

Fuster, J.M., 1997. Network memory. Trends Neurosci. 20, 451-459. https://doi.org/ 10.1016/S0166-2236(97)01128-4.

Gilboa, A., Marlatte, H., 2017. Neurobiology of schemas and schema-mediated memory. Trends Cognit. Sci. 21, 618-631. https://doi.org/10.1016/j.tics.2017.04.013.

Grill-Spector, K., Henson, R., Martin, A., 2006. Repetition and the brain: neural models of stimulus-specific effects. Trends Cognit. Sci. 10, 14-23. https://doi.org/10.1016/ j.tics.2005.11.006.

Grotheer, M., Kovács, G., 2016. Can predictive coding explain repetition suppression? Cortex 80, 113-124. https://doi.org/10.1016/j.cortex.2015.11.027.

Guntupalli, J.S., Hanke, M., Halchenko, Y.O., Connolly, A.C., Ramadge, P.J., Haxby, J.V., 2016. A model of representational spaces in human. Cortex. Cereb. Cortex 26 , 2919-2934. https://doi.org/10.1093/cercor/bhw068.

Gusnard, D.A., Raichle, M.E., 2001. Searching for a baseline: functional imaging and the resting human brain. Nat. Rev. Neurosci. 2, 685-694. https://doi.org/10.1038/ 35094500 .

Habib, R., Nyberg, L., Tulving, E., 2003. Hemispheric asymmetries of memory: the HERA model revisited. Trends Cognit. Sci. 7, 241-245. https://doi.org/10.1016/S13646613(03)00110-4.

Hassabis, D., Maguire, E.A., 2007. Deconstructing episodic memory with construction. Trends Cognit. Sci. 11, 299-306. https://doi.org/10.1016/j.tics.2007.05.001.

Hasson, U., Chen, J., Honey, C.J., 2015. Hierarchical process memory: memory as an integral component of information processing. Trends Cognit. Sci. 19, 304-313. https://doi.org/10.1016/j.tics.2015.04.006.

Hasson, U., Malach, R., Heeger, D.J., 2010. Reliability of cortical activity during natural stimulation. Trends Cognit. Sci. 14, 40-48. https://doi.org/10.1016/ j.tics.2009.10.011.

Hasson, U., Nir, Y., Levy, I., Fuhrmann, G., Malach, R., 2004. Intersubject synchronization of cortical activity during natural vision. Science 303, 1634-1640. https://doi.org/ $10.1126 /$ science. 1089506.

Haxby, J.V., 2001. Distributed and overlapping representations of faces and objects in ventral temporal cortex. Science (80-. ) 293, 2425-2430. https://doi.org/10.1126/ science. 1063736.

Hayama, H.R., Vilberg, K.L., Rugg, M.D., 2012. Overlap between the neural correlates of cued recall and source memory: evidence for a generic recollection network? J. Cogn. Neurosci 24, 1127-1137. https://doi.org/10.1162/jocn_a 00202.

Hupbach, A., Gomez, R., Hardt, O., Nadel, L., 2007. Reconsolidation of episodic memories: a subtle reminder triggers integration of new information. Learn. Mem. 14, 47-53. https://doi.org/10.1101/lm.365707.

Jääskeläinen, I.P., Koskentalo, K., Balk, M.H., Autti, T., Kauramäki, J., Pomren, C., Sams, M., 2008. Inter-subject synchronization of prefrontal cortex hemodynamic activity during natural viewing. Open Neuroimag. J. 2, 14-19. https://doi.org/ 10.2174/1874440000802010014.

Jenkinson, M., Beckmann, C.F., Behrens, T.E.J., Woolrich, M.W., Smith, S.M., 2012. FSL. Neuroimage 62, 782-790. https://doi.org/10.1016/j.neuroimage.2011.09.015.

Johnson, J.D., Suzuki, M., Rugg, M.D., 2013. Recollection, familiarity, and contentsensitivity in lateral parietal cortex: a high-resolution fMRI study. Front. Hum. Neurosci. 7, 1-15. https://doi.org/10.3389/fnhum.2013.00219.

Jung-Beeman, M., 2005. Bilateral brain processes for comprehending natural language. Trends Cognit. Sci. 9, 512-518. https://doi.org/10.1016/j.tics.2005.09.009.

Kauppi, J.-P., Jääskeläinen, I.P., Sams, M., Tohka, J., 2010. Inter-subject correlation of brain hemodynamic responses during watching a movie: localization in space and frequency. Front. Neuroinform 4, 5. https://doi.org/10.3389/fninf.2010.00005.

Kauttonen, J., Hlushchuk, Y., Hasson, U., Jääskeläinen, I, Tikka, P., 2015, Neural functions of memory retrieval and narrative reconstruction during free-viewing of film Memento. In: Annual Meeting of the Society for Neuroscience. Chicago.

Kauttonen, J., Kaipainen, M., Tikka, P., 2014. Model of narrative nowness for neurocinematic experiments. In: 5th Workshop on Computational Models of Narrative. pp. 77-87. https://doi.org/10.4230/OASIcs.CMN.2014.77.

Kim, H., 2013. Differential neural activity in the recognition of old versus new events: an Activation Likelihood Estimation Meta-Analysis. Hum. Brain Mapp. 34, 814-836. https://doi.org/10.1002/hbm.21474.

Kim, H., 2011. Neural activity that predicts subsequent memory and forgetting: a metaanalysis of 74 fMRI studies. Neuroimage 54, 2446-2461. https://doi.org/10.1016/ j.neuroimage.2010.09.045. 
Kim, H., 2010. Dissociating the roles of the default-mode, dorsal, and ventral networks in episodic memory retrieval. Neuroimage 50, 1648-1657. https://doi.org/10.1016/ j.neuroimage.2010.01.051.

King-Casas, B., Tomlin, D., Anen, C., Camerer, C.F., Quartz, S.R., Montague, P.R., 2005 Getting to know you: reputation and trust in a two-person economic exchange. Science 308, 78-83. https://doi.org/10.1126/science.1108062.

Kriegeskorte, N., Goebel, R., Bandettini, P., 2006. Information-based functional brain mapping. Proc. Natl. Acad. Sci. U. S. A 103, 3863-3868. https://doi.org/10.1073/ pnas.0600244103.

Kuhl, B.A., Chun, M.M., 2014. Successful remembering elicits event-specific activity patterns in lateral parietal cortex. J. Neurosci. 34, 8051-8060. https://doi.org/ 10.1523/JNEUROSCI.4328-13.2014.

Kumaran, D., Summerfield, J.J., Hassabis, D., Maguire, E.A., 2009. Tracking the emergence of conceptual knowledge during human decision making. Neuron 63, 889-901. https://doi.org/10.1016/j.neuron.2009.07.030.

Lahnakoski, J.M., Glerean, E., Jääskeläinen, I.P., Hyönä, J., Hari, R., Sams, M., Nummenmaa, L., 2014. Synchronous brain activity across individuals underlies shared psychological perspectives. Neuroimage 100, 316-324. https://doi.org/ 10.1016/j.neuroimage.2014.06.022.

Lahnakoski, J.M., Salmi, J., Jääskeläinen, I.P., Lampinen, J., Glerean, E., Tikka, P., Sams, M., 2012. Stimulus-related independent component and voxel-wise analysis of human brain activity during free viewing of a feature film. PLoS One 7, e35215. https://doi.org/10.1371/journal.pone.0035215.

Lerner, Y., Honey, C.J., Silbert, L.J., Hasson, U., 2011. Topographic mapping of a hierarchy of temporal receptive windows using a narrated story. J. Neurosci. 31, 2906-2915. https://doi.org/10.1523/JNEUROSCI.3684-10.2011.

Lu, K.-H., Hung, S.-C., Wen, H., Marussich, L., Liu, Z., Drury, H., 2016. Influences of highlevel features, gaze, and scene transitions on the reliability of BOLD responses to natural movie stimuli. PLoS One 11, e0161797. https://doi.org/10.1371/ journal.pone.0161797.

Maguire, E.A., Frith, C.D., Morris, R.G., 1999. The functional neuroanatomy of comprehension and memory: the importance of prior knowledge. Brain 122 (Pt 1), 1839-1850.

Mar, R.A., 2004. The neuropsychology of narrative: story comprehension, story production and their interrelation. Neuropsychologia 42, 1414-1434. https:// doi.org/10.1016/j.neuropsychologia.2003.12.016.

Marini, A., Carlomagno, S., Caltagirone, C., Nocentini, U., 2005. The role played by the right hemisphere in the organization of complex textual structures. Brain Lang. 93, 46-54. https://doi.org/10.1016/j.bandl.2004.08.002.

McKenzie, S., Eichenbaum, H., 2011. Consolidation and reconsolidation: two lives of memories? Neuron 71, 224-233. https://doi.org/10.1016/j.neuron.2011.06.037.

Mourão-Miranda, J., Reynaud, E., McGlone, F., Calvert, G., Brammer, M., 2006. The impact of temporal compression and space selection on SVM analysis of single-subject and multi-subject fMRI data. Neuroimage 33, 1055-1065. https://doi.org/10.1016/ j.neuroimage.2006.08.016.

Naci, L., Cusack, R., Anello, M., Owen, A.M., 2014. A common neural code for similar conscious experiences in different individuals. Proc. Natl. Acad. Sci. Unit. States Am. 111, 14277-14282. https://doi.org/10.1073/pnas.1407007111.

Nadel, L., Hardt, O., 2011. Update on memory systems and processes. Neuropsychopharmacology 36, 251-273. https://doi.org/10.1038/npp.2010.169.

Nili, H., Wingfield, C., Walther, A., Su, L., Marslen-Wilson, W., Kriegeskorte, N., 2014 A toolbox for representational similarity analysis. PLoS Comput. Biol. 10, e1003553 https://doi.org/10.1371/journal.pcbi.1003553.

Norman, K., Polyn, S.M., Detre, G.J., Haxby, J.V., 2006. Beyond mind-reading: multivoxel pattern analysis of fMRI data. Trends Cognit. Sci. 10, 424-430. https://doi.org/ 10.1016/j.tics.2006.07.005.

Oedekoven, C.S.H., Keidel, J.L., Berens, S.C., Bird, C.M., 2017. Reinstatement of memory representations for lifelike events over the course of a week. Sci. Rep. 7, 14305. https://doi.org/10.1038/s41598-017-13938-4.

Polyn, S.M., Natu, V.S., Cohen, J.D., Norman, K., 2005. Category-specific cortical activity precedes retrieval during memory search. Science 310, 1963-1966. https://doi.org/ 10.1126/science.1117645.

Power, J.D., Barnes, K., Snyder, A.Z., Schlaggar, B.L., Petersen, S.E., 2012. Spurious but systematic correlations in functional connectivity MRI networks arise from subject motion. Neuroimage 59, 2142-2154. https://doi.org/10.1016/ j.neuroimage.2011.10.018.

Preston, A.R., Eichenbaum, H., 2013. Interplay of hippocampus and prefrontal cortex in memory. Curr. Biol. 23, R764-R773. https://doi.org/10.1016/j.cub.2013.05.041.

Price, C.J., 2012. A review and synthesis of the first 20 years of PET and fMRI studies of heard speech, spoken language and reading. Neuroimage 62, 816-847. https:// doi.org/10.1016/j.neuroimage.2012.04.062.

Reggev, N., Bein, O., Maril, A., 2016. Distinct Neural Suppression and Encoding Effects for Conceptual Novelty and Familiarity, pp. 1455-1470. https://doi.org/10.1162/ jocn.

Richter, F.R., Chanales, A.J.H., Kuhl, B.A., 2016. Predicting the integration of overlapping memories by decoding mnemonic processing states during learning. Neuroimage 124, 323-335. https://doi.org/10.1016/j.neuroimage.2015.08.051.

Rugg, M.D., King, D.R., 2017. ScienceDirect Special issue : review Ventral lateral parietal cortex and episodic memory retrieval. Cortex 1-13. https://doi.org/10.1016/ j.cortex.2017.07.012.

Rugg, M.D., Vilberg, K.L., 2013. Brain networks underlying episodic memory retrieval. Curr. Opin. Neurobiol. 23, 255-260. https://doi.org/10.1016/j.conb.2012.11.005.

Saarimäki, H., Gotsopoulos, A., Jääskeläinen, I.P., Lampinen, J., Vuilleumier, P., Hari, R., Sams, M., Nummenmaa, L., 2016. Discrete neural signatures of basic emotions. Cerebr. Cortex 26, 2563-2573. https://doi.org/10.1093/cercor/bhv086.
Scalici, F., Caltagirone, C., Carlesimo, G.A., 2017. The contribution of different prefrontal cortex regions to recollection and familiarity. A review of fMRI data. Neurosci. Biobehav. Rev. 83, 240-251. https://doi.org/10.1016/j.neubiorev.2017.10.017.

Schapiro, A.C., Rogers, T.T., Cordova, N.I., Turk-Browne, N.B., Botvinick, M.M., 2013. Neural representations of events arise from temporal community structure. Nat. Neurosci. 16, 486-492. https://doi.org/10.1038/nn.3331.

Schlichting, M.L., Mumford, J.A., Preston, A.R., 2015. Learning-related representational changes reveal dissociable integration and separation signatures in the hippocampus and prefrontal cortex. Nat. Commun. 6, 1-10. https://doi.org/10.1038/ ncomms9151.

Schlichting, M.L., Preston, A.R., 2015. Memory integration: neural mechanisms and implications for behavior. Curr. Opin. Behav. Sci. 1, 1-8. https://doi.org/10.1016/ j.cobeha.2014.07.005.

Schon, K., Ross, R.S., Hasselmo, M.E., Stern, C.E., 2013. Complementary roles of medial temporal lobes and mid-dorsolateral prefrontal cortex for working memory for novel and familiar trial-unique visual stimuli. Eur. J. Neurosci. 37, 668-678. https:// doi.org/10.1111/ejn.12062.

Segaert, K., Weber, K., de Lange, F.P., Petersson, K.M., Hagoort, P., 2013. The suppression of repetition enhancement: a review of fMRI studies. Neuropsychologia 51, 59-66. https://doi.org/10.1016/j.neuropsychologia.2012.11.006.

Sharon, T., Moscovitch, M., Gilboa, A., 2011. Rapid neocortical acquisition of long-term arbitrary associations independent of the hippocampus. Proc. Natl. Acad. Sci. U. S. A 108, 1146-1151. https://doi.org/10.1073/pnas.1005238108.

Skerry, A.E., Saxe, R., 2014. A common neural code for perceived and inferred emotion, 34, 15997-16008. https://doi.org/10.1523/JNEUROSCI.1676-14.2014.

St-Laurent, M., Abdi, H., Buchsbaum, B.R., 2015. Distributed patterns of reactivation predict vividness of recollection. J. Cogn. Neurosci 1-19. https://doi.org/10.1162/ jocn_a_00839.

St George, M., Kutas, M., Martinez, A., Sereno, M.I., 1999. Semantic integration in reading: engagement of the right hemisphere during discourse processing. Brain 122 (Pt 7), 1317-1325.

Summerfield, J.J., Lepsien, J., Gitelman, D.R., Mesulam, M.M., Nobre, A.C., 2006. Orienting attention based on long-term memory experience. Neuron 49, 905-916. https://doi.org/10.1016/j.neuron.2006.01.021.

Thakral, P.P., Wang, T.H., Rugg, M.D., 2017. Decoding the content of recollection within the core recollection network and beyond. Cortex 91, 101-113. https://doi.org/ 10.1016/j.cortex.2016.12.011.

Thakral, P.P., Wang, T.H., Rugg, M.D., 2015. Cortical reinstatement and the confidence and accuracy of source memory. Neuroimage 109, 118-129. https://doi.org/ 10.1016/j.neuroimage.2015.01.003.

Tikka, P., 2008. Enactive Cinema: Simulatorium Eisensteinense.

Tomlin, D., Kayali, M.A., King-Casas, B., Anen, C., Camerer, C.F., Quartz, S.R., Montague, P.R., 2006. Agent-specific responses in the cingulate cortex during economic exchanges. Science 312, 1047-1050. https://doi.org/10.1126/ science.1125596.

Tulving, E., Kapur, S., Craik, F.I., Moscovitch, M., Houle, S., 1994. Hemispheric encoding/ retrieval asymmetry in episodic memory: positron emission tomography findings. Proc. Natl. Acad. Sci. Unit. States Am. 91, 2016-2020. https://doi.org/10.1073/ pnas.91.6.2016.

Tylén, K., Christensen, P., Roepstorff, A., Lund, T., Østergaard, S., Donald, M., 2015. Brains striving for coherence: long-term cumulative plot formation in the default mode network. Neuroimage 121, 106-114. https://doi.org/10.1016/ j.neuroimage.2015.07.047.

Utevsky, A.V., Smith, D.V., Huettel, S., 2014. Precuneus is a functional core of the defaultmode network. J. Neurosci. 34, 932-940. https://doi.org/10.1523/ JNEUROSCI.4227-13.2014.

van Kesteren, M.T.R., Beul, S.F., Takashima, A., Henson, R.N., Ruiter, D.J., Fernández, G., 2013. Differential roles for medial prefrontal and medial temporal cortices in schemadependent encoding: from congruent to incongruent. Neuropsychologia 51, 2352-2359. https://doi.org/10.1016/j.neuropsychologia.2013.05.027.

van Kesteren, M.T.R., Fernández, G., Norris, D.G., Hermans, E.J., 2010. Persistent schemadependent hippocampal-neocortical connectivity during memory encoding and postencoding rest in humans. Proc. Natl. Acad. Sci. U. S. A 107, 7550-7555. https:// doi.org/10.1073/pnas.0914892107.

van Kesteren, M.T.R., Rijpkema, M., Ruiter, D.J., Morris, R.G.M., Fernández, G., 2014. Building on prior knowledge: schema-dependent encoding processes relate to academic performance. J. Cogn. Neurosci 26, 2250-2261. https://doi.org/10.1162/ jocn_a_00630.

Vilberg, K.L., Rugg, M.D., 2008. Memory retrieval and the parietal cortex: a review of evidence from a dual-process perspective. Neuropsychologia 46, 1787-1799. https:// doi.org/10.1016/j.neuropsychologia.2008.01.004.

Vincent, J.L., Kahn, I., Snyder, A.Z., Raichle, M.E., Buckner, R.L., 2008. Evidence for a frontoparietal control system revealed by intrinsic functional connectivity. J. Neurophysiol. 100, 3328-3342. https://doi.org/10.1152/jn.90355.2008.

Vodrahalli, K., Chen, P.H., Liang, Y., Baldassano, C., Chen, J., Yong, E., Honey, C., Hasson, U., Ramadge, P., Norman, K.A., Arora, S., 2017. Mapping between fMRI responses to movies and their natural language annotations. Neuroimage 1-9. https://doi.org/10.1016/j.neuroimage.2017.06.042.

Wake, S.J., Izuma, K., 2017. A Common Neural Code for Social and Monetary Rewards in the Human Striatum, pp. 1558-1564. https://doi.org/10.1093/scan/nsx092.

Walther, A., Nili, H., Ejaz, N., Alink, A., Kriegeskorte, N., Diedrichsen, J., 2016. Reliability of dissimilarity measures for multi-voxel pattern analysis. Neuroimage 137, 188-200. https://doi.org/10.1016/j.neuroimage.2015.12.012.

Wheeler, M.A., Stuss, D.T., Tulving, E., 1995. Frontal lobe damage produces episodic memory impairment. J. Int. Neuropsychol. Soc. 1, 525. https://doi.org/10.1017/ S1355617700000655. 
Whitney, C., Huber, W., Klann, J., Weis, S., Krach, S., Kircher, T., 2009. Neural correlates of narrative shifts during auditory story comprehension. Neuroimage 47, 360-366. https://doi.org/10.1016/j.neuroimage.2009.04.037.

Winkler, A.M., Ridgway, G.R., Webster, M.A., Smith, S.M., Nichols, T.E., 2014. Permutation inference for the general linear model. Neuroimage 92, 381-397. https://doi.org/10.1016/j.neuroimage.2014.01.060.

Xiao, X., Dong, Q., Gao, J., Men, W., Poldrack, R.A., Xue, G., 2017. Transformed neural pattern reinstatement during episodic memory retrieval. J. Neurosci. 37, 2986-2998. https://doi.org/10.1523/JNEUROSCI.2324-16.2017.
Xu, J., Kemeny, S., Park, G., Frattali, C., Braun, A., 2005. Language in context: emergent features of word, sentence, and narrative comprehension. Neuroimage 25, 1002-1015. https://doi.org/10.1016/j.neuroimage.2004.12.013.

Yaffe, R.B., Kerr, M.S.D., Damera, S., Sarma, S.V., Inati, S.K., Zaghloul, K., 2014. Reinstatement of distributed cortical oscillations occurs with precise spatiotemporal dynamics during successful memory retrieval. Proc. Natl. Acad. Sci. U. S. A 111, 18727-18732. https://doi.org/10.1073/pnas.1417017112. 